\title{
Anatomical Variations of the Peroneus Tertius and Extensor Digitorum Longus Muscles - Case Presentation
}

\author{
Mihai-luliu Harșa1, Loránd Kocsis', Angéla-Mária Czundel', Lóránd Dénes², Zsuzsanna Pap² \\ 1 "George Emil Palade" University of Medicine, Pharmacy, Science and Technology, Târgu Mureș, Romania \\ 2 Department of Anatomy and Embryology, "George Emil Palade" University of Medicine, Pharmacy, Science and Technology, Târgu \\ Mureș, Romania
}

\section{CORRESPONDENCE \\ Loránd Kocsis \\ Str. Gheorghe Marinescu nr. 38 \\ 540139 Târgu Mureș, Romania \\ Tel: +40 745549232 \\ E-mail: Iorand.kocsis@umfst.ro}

\section{ARTICLE HISTORY}

Received: April 8, 2021

Accepted: April 16, 2021
Mihai-Iuliu Harșa • Str. Gheorghe Marinescu nr. 38 540139 Târgu Mureș, Romania. Tel: +40 745549 232, E-mail: mihai-iuliu.harsa@umfst.ro

Angéla-Mária Czundel • Str. Gheorghe Marinescu nr. 38, 540139 Târgu Mures, Romania. Tel: +40 745549 232, E-mail: czundel_angela@yahoo.com

Lóránd Dénes • Str. Gheorghe Marinescu nr. 38 540139 Târgu Mureș, Romania. Tel: +40 745549 232, E-mail: deneslorand@gmail.com

Zsuzsanna Pap • Str. Gheorghe Marinescu nr. 38, 540139 Târgu Mures, Romania. Tel: +40 265215 551, E-mail: zsuzsanna.pap@umfst.ro

\begin{abstract}
Introduction: The extensor digitorum longus and peroneus tertius muscles have multiple morphological variations. The tendinous slips of these muscles are used as grafts to replace other damaged tendons. Case presentation: We present a unique anatomical variant observed during dissection of a cadaver at the Department of Anatomy and Embryology of the "George Emil Palade" University of Medicine, Pharmacy, Science and Technology of Târgu Mureș, Romania. The peroneus tertius muscle was identified on the left lower extremity as originating from the inferior third of the fibula and interosseus membrane. The posterior belly of this muscle joins the muscular belly of the extensor digitorum longus muscle. The tendons of toes II to $\mathrm{V}$ detach from the extensor digitorum longus muscle with an additional tendon. This last tendon gives off an accessory tendinous slip; lateral to this, the peroneus tertius muscle tendon is observed. In case of the right lower limb we also noted the peroneus tertius muscle originating from the distal part of the extensor digitorum longus muscle. Regarding the extensor digitorum longus muscle we describe an accessory tendinous slip. Conclusion: The anatomical variation reported in this study is similar to those reported by previous studies, but displays particular characteristics regarding the $\mathrm{VI}$ tendon of extensor digitorum longus and the joining of the peroneus tertius and extensor digitorum longus muscles in the left lower extremity.
\end{abstract}

Keywords: extensor digitorum longus muscle, peroneus tertius muscle, anatomical variations

\section{INTRODUCTION}

The anterior compartment of the leg is limited on one side by the osteofibrous plane of the medially placed tibia, the laterally placed fibula, and the interosseous membrane that attaches onto the interosseous crests of the diaphysis, and closes the interosseous space between the tibia and fibula; the other limiting structure is the anterior intermuscular septum that detaches from the crural fascia and inserts onto the anterior margin of the fibula and lateral margin of the tibia. The muscles of the anterior compartment of the 


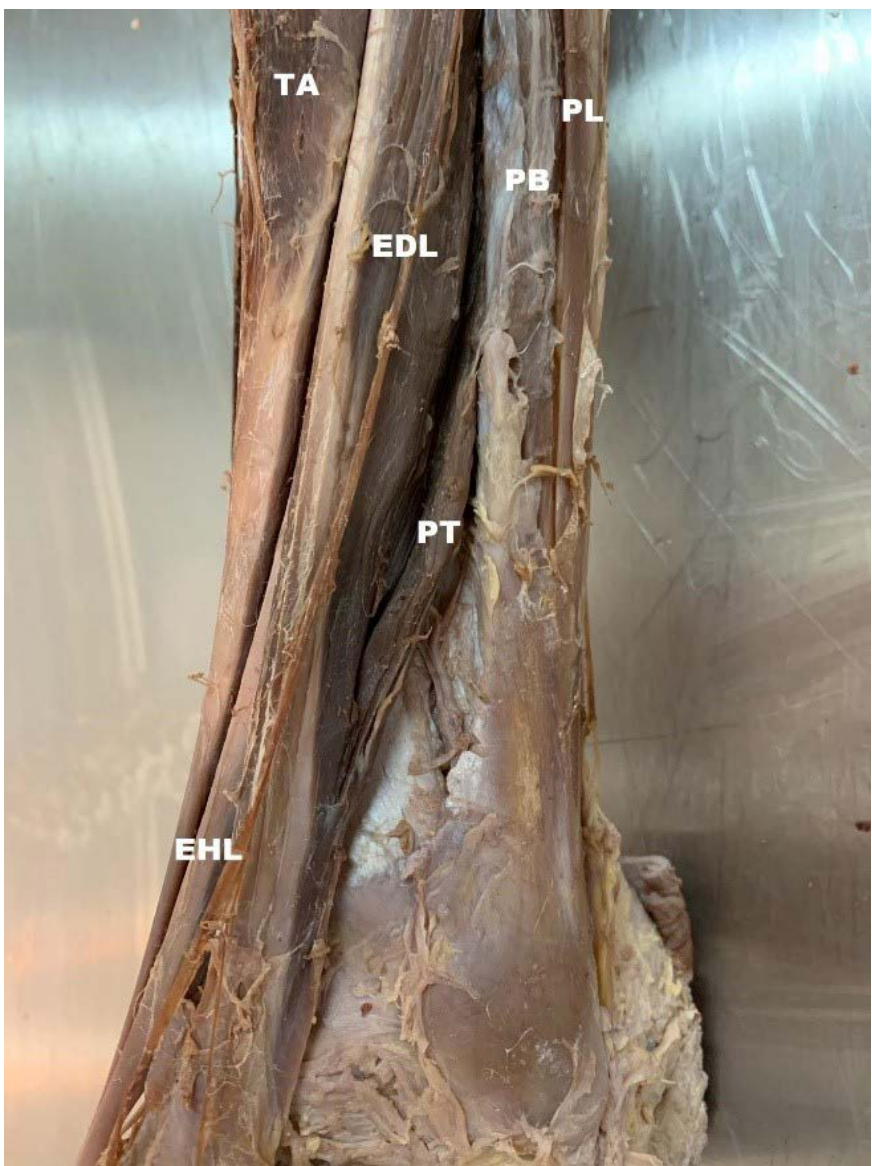

FIGURE 1. The muscle belly of the peroneus tertius muscle joins the muscle belly of the extensor digitorum longus muscle, left lower extremity. PT - musculus peroneus tertius; EDL - musculus extensor digitorum longus; $\mathrm{EHL}$ - tendon of musculus extensor hallucis longus; TA - musculus tibialis anterior; PB - musculus peroneus brevis; $\mathrm{PL}$ - musculus peroneus longus

leg are listed in medio-lateral direction: musculus tibialis anterior (TA), musculus extensor hallucis longus (EHL), musculus extensor digitorum longus (EDL), and musculus peroneus tertius (PT). According to anatomy textbooks, TA comes from the lateral condyle and upper half of the lateral surface of the tibia, and the neighboring surface of the interosseous membrane and crural fascia; it inserts onto the plantar surface of the medial cuneiform. EHL originates from the middle part of the fibular medial surface and the neighboring interosseous membrane; it inserts onto the base of the distal phalanx of the halux through tendon I. EDL comes from the lateral condyle of the tibia, the proximal $2 / 3$ of the anterior margin of the fibula, the superior part of the interosseous membrane, the deep fascia of the region and the anterior intermuscular septum; it inserts through fibrous expansions of tendons II-V onto the middle and distal phalanx of fingers II-V.1,2

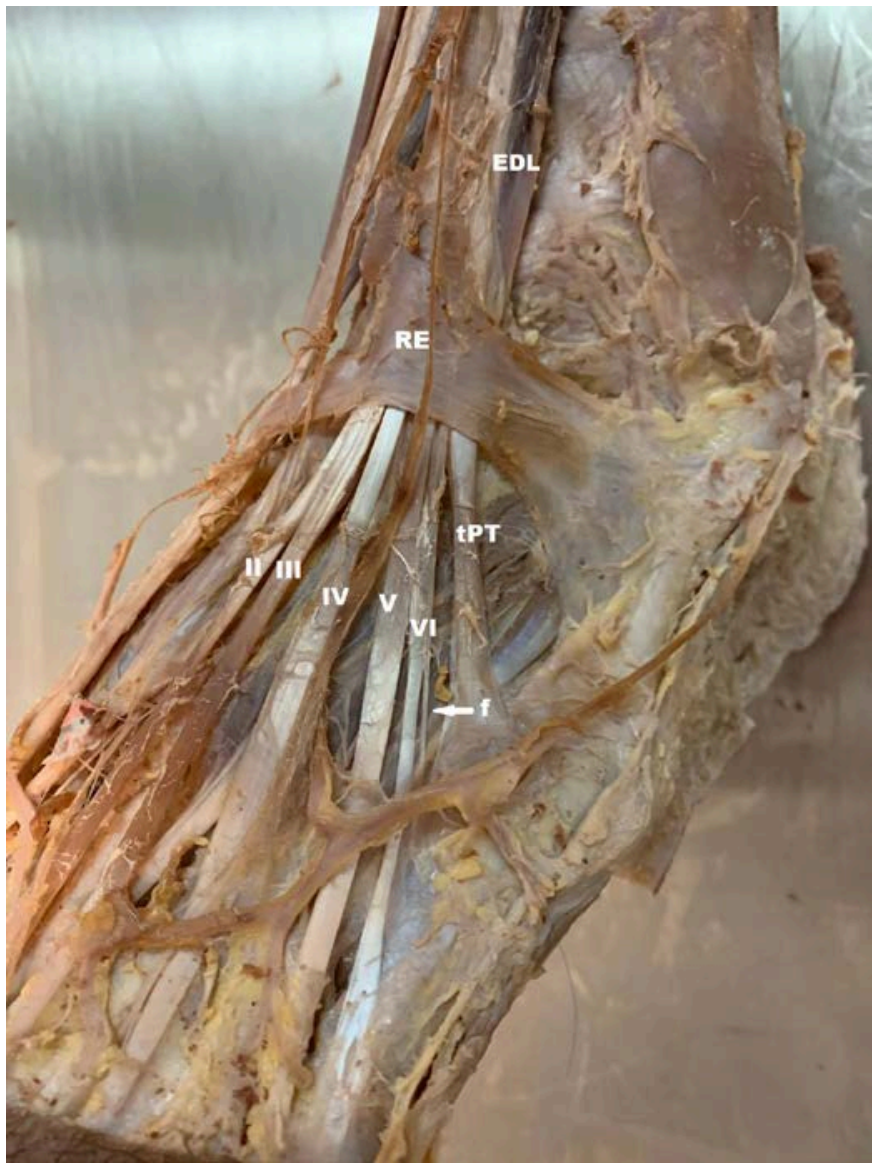

FIGURE 2. Tendon VI of EDL with its detached tendon slip (f), left lower extremity. RE - retinaculum extensorum, EDL - musculus extensor digitorum longus; tPT - tendon of musculus peroneus tertius

EDL has the following topographical relationships on the leg: the anterior surface with the fascia of the leg and skin; medially with the tibialis anterior (TA) muscle and the extensor hallucis longus (EHL) muscle, as well as with the anterior tibial vasculo-nervous bundle; laterally through the anterior intermuscular septum with the peroneus longus (PL) and peroneus brevis (PB) muscles. EDL is innervated by branches of the deep fibular nerve. It produces extension of fingers II-V and dorsal flexion of the foot, with an additional external rotation (pronation). ${ }^{2}$

$\mathrm{PT}$ originates from the distal part of the fibula, the interosseus membrane, and anterior intermuscular septum; it inserts through a tendon (tendon VI) on the dorsomedial surface of the base of metatarsal $\mathrm{V}$, and also the medial surface of this bone, through a thin expansion. It is often described as part of EDL. ${ }^{3}$

According to published data, the bellies and tendons of EDL and PT have numerous variations. Knowledge and identification of these variations is an advantage for orthopedic surgeons and plastic surgery surgeons; the addition- 


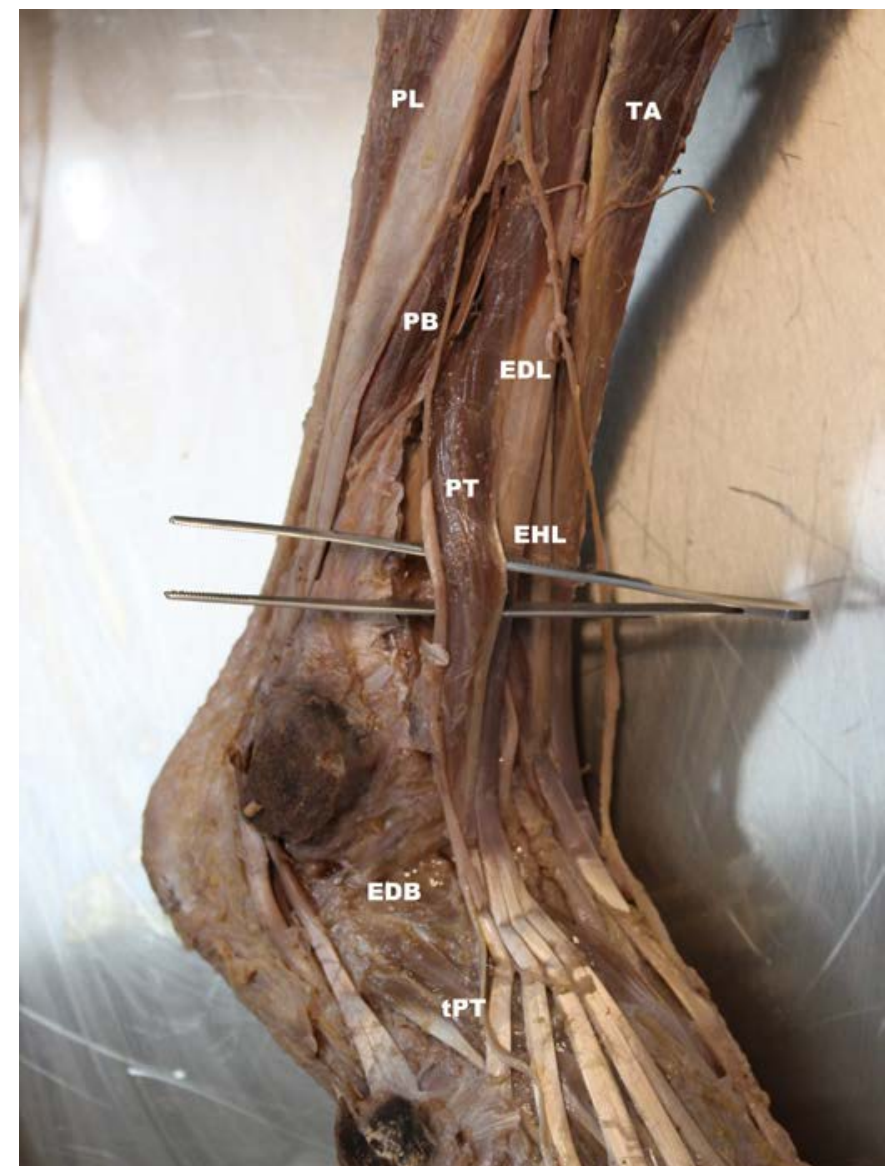

FIGURE 3. Separation of the PT muscle belly from EDL, right lower extremity. tPT - tendon of musculus peroneus tertius; PT musculus peroneus tertius; tPT - tendon of musculus peroneus tertius; EDL - musculus extensor digitorum longus; ELH - tendon of musculus hallucis longus; EDB - musculus extensor digitorum brevis; TA - musculus tibialis anterior; $\mathrm{PL}$ - musculus peroneus longus; PB - musculus peroneus brevis

al tendon slips are potential graft sources for replacement of damaged tendons. ${ }^{4}$

\section{CASE PRESENTATION}

Didactic dissection of a male cadaver has been performed at the Department of Anatomy and Embryology of the "George Emil Palade" University of Medicine, Pharmacy, Science and Technology of Târgu Mureș, Romania, and multiple variations of the EDL and PT muscles have been identified.

On the left lower extremity, a muscle bundle has been identified as the PT muscle, originating from the inferior third of the fibula, the interosseous membrane, and the anterior intermuscular septum. After descending for about $10 \mathrm{~cm}$, this bundle joins the muscle belly of the EDL muscle to create a common muscle body (Figure 1).

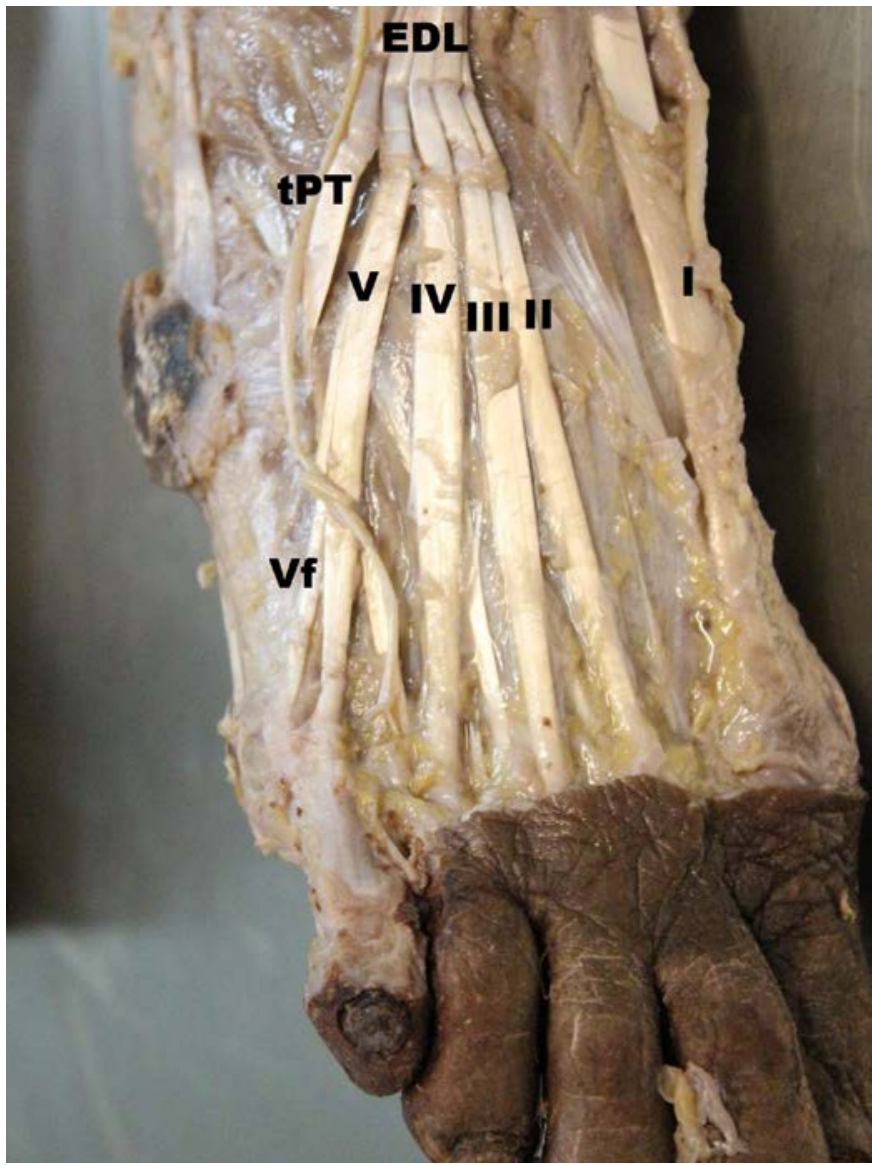

FIGURE 4. The tendon slip (Vf) detached from tendon $\mathrm{V}$ of EDL, right lower extremity. tPT - tendon of musculus peroneus tertius; $\mathrm{EDL}$ - tendon of musculus extensor digitorum longus; I - tendon of musculus extensor hallucis longus

At the extensor retinaculum (RE) six tendons and a tendon slip detach from the common EDL (Figure 2). The first four tendons (II-V) correspond to the classical description of EDL tendons. Therefore, tendons II-V insert onto the middle and distal phalanx of the corresponding toes II to V. Tendon VI inserts onto the joint capsule of the 5th metatarsophalangeal joint, and the accessory tendon slip (f) detached from this tendon inserts onto the dorsal aponeurosis of the 5th toe. The tendon of the peroneus tertius muscle (tPT) inserts onto the dorsomedial surface of metatarsal $\mathrm{V}$, according to the classical description (Figure 2).

On the right lower extremity, a PT muscle has been identified originating from the distal part of the EDL muscle belly, with a subsequent course that matches the one described in the literature. Its tendon (tPT) inserts onto the base of metatarsal V (Figure 3). EDL has four tendons (II-V) that insert onto the middle and distal phalanx of the corresponding toes II to $\mathrm{V}^{3}$ An accessory tendon slip (Vf) 
detaches from tendon $V$ and inserts of the joint capsule of the 5th metatarsophalangeal joint (Figure 4).

\section{DISCUSSIONS}

In this case study we describe an asymmetrical anatomical variation of the EDL and PT muscles. On the left lower extremity, we observed a muscle bundle identified as the PT muscle, originating from the inferior third of the fibula, the interosseous membrane and the anterior intermuscular septum. Following the descending course of this muscle bundle we observed that its muscle fibers join the muscular belly of the EDL muscle.

Therefore, this common muscle belly $(\mathrm{EDL}+\mathrm{PT})$ provided the following tendons in medio-lateral order: tendon II inserted onto the middle and distal phalanx of the 2 nd toe, tendon III inserts onto the middle and distal phalanx of the 3rd toe, tendon IV inserted onto the middle and distal phalanx of the 4th toe, tendon $\mathrm{V}$ inserted onto the middle and distal phalanx of the 5th toe, tendon VI inserted onto the joint capsule of the 5th metatarsophalangeal joint, and the accessory tendon slip of this tendon (f) inserted onto the dorsal aponeurosis of the 5 th toe, while tPT inserted onto the dorsomedial surface of the 5th metatarsal's base.

On the right lower extremity, we observed a PT muscle originating from the distal part of the EDL muscle belly. Its tendon (tPT) inserted onto the base of the 5th metatarsal, according to classical descriptions of insertion characteristics for PT. A tendon slip (Vf) detached from tendon $\mathrm{V}$ of the EDL muscle and inserted onto the capsule of the 5th metatarsophalangeal joint. Insertion of the remaining tendons (II-V) was identical to that observed on the left lower extremity.

Several anatomical variations of the EDL muscle insertion have been reported. ${ }^{5-7,10,11}$ Just like in our case, numerous bifurcation variations and additional tendon slips are discussed. In these cases, the bifurcated tendons and tendon slips insert onto their corresponding toes. In our case, we observed on the right lower extremity the bifurcated insertion of tendon $\mathrm{V}$, along with an accessory tendon slip (Vf) that also inserted onto the 5th toe. ${ }^{78}$ More precisely, this accessory tendon slip inserted onto the joint capsule of the 5th metatarsophalangeal joint. Berman et al. reported accessory tendon slips inserting onto the metatarsals that correspond to the classical tendons (II-V), onto the EDL tendons or onto the dorsal aponeurosis of the fingers. ${ }^{7}$ There have been case reports where EDL tendons of one or more toes were missing or an additional tendon was described. Apart from these findings, in our case, we observed an additional tendon (VI) on the left lower extremity, which inserted onto the joint capsule of the metatarsophalangeal joint of the 5th toe. This additional tendon presented a tendon slip (f) inserting on the dorsal aponeurosis of the 5th toe, which has not been reported so far. No similar variants were found in the literature.

In some cases, enlargement of PT has been reported, and if this was missing, then compensatory hypertrophy of the EDL muscle may have been present. ${ }^{9-14}$

PT may be present in over $90 \%$ of the cases, but recent studies suggest a lower prevalence (49.1-81.5\%). The PT muscle is characteristic to humans; it may have different sizes and plays a role in bipedal movements. ${ }^{15-17}$

Only a small number of studies deal with anatomical variations of the PT muscle. Rourke et al. described a particular variation of the PT muscle, originating as a continuation of the EDL muscle, similar to the right lower extremity of our case. An interesting aspect of this study was the asymmetry between the PT muscles of the same cadaver. ${ }^{18}$ PT muscle asymmetry was also present in our case.

The left lower extremity has the most frequent variants of the PT muscle, ${ }^{3}$ with proper origin and classical insertion, but the particular aspect of our case was the joining of the PT and ELD muscle bellies. This variation was presented in only one previously reported case. ${ }^{19}$ The right lower extremity presented a more rare variant, which has already been reported by other authors, ${ }^{3,6}$ originating from the distal part of the EDL muscle belly, but with classical course and insertion.

Yildiz et al. have described a unique variation. The EHL muscle belly bifurcated at $17 \mathrm{~cm}$ from its origin into a lateral and a medial muscle bundle. The lateral muscle bundle inserted onto the base of the 5th metatarsal and has been identified by the authors as the PT muscle. ${ }^{20}$ Apart from the variation described earlier, we did not find variations of the EHL in both lower extremities, nor variations of the EHL which interfere with the EDL or PT muscles.

The most particular findings in our study on the left lower extremity, were the 6th tendon of the EDL and its accessory tendinous slip, along with the joining of the PT and EDL muscle bellies. Other least common findings described by other authors include the bifurcation of the 5th tendon of EDL and the origin of PT from the distal half of EDLs' muscle belly on the right lower extremity. ${ }^{18,20}$

Numerous studies have emphasized the importance of the PT muscle in medical practice, especially in plastic surgery, considering its frequent use as a graft (island flap). It has good viability due to numerous anastomoses of perforating branches of the fibular, malleolar and lateral tarsal arteries. ${ }^{21}$ 


\section{CONCLUSIONS}

Anatomical variations of shape, origin and insertion of musculus extensor digitorum longus and peroneus tertius have been reported in publications with clinical implications in the field of orthopedic surgery and plastic surgery. The particular aspect of our case, which has not yet been reported, was the fact that the muscle belly of musculus peroneus tertius joined up with the belly of musculus extensor digitorum longus.

\section{CONFLICT OF INTEREST}

Nothing to declare.

\section{ETHICAL CONSIDERATIONS}

The case presentation and the use of cadavers for scientific purposes was approved by the Ethics Committee of the "George Emil Palade" University of Medicine, Pharmacy, Science and Technology of Târgu Mureș, Romania $(1323 / 04.2021)$.

\section{REFERENCES}

1. Seres-Sturm L, Pávai Z, Seres-Sturm M, Dénes L. A végtagok anatómiája. Târgu Mureș: University Press, 2005; p. 177-178.

2. Enciulescu C. Anatomia Membrelor. Târgu Mureş: University Press, 2015; p. 82-83.
3. Gray H, Carter HV. Grays Anatomy. London: Arcturus, 2019; p. 1406-1408.

4. Ramirez D, Gajardo C, Caballero P. Clinical Evaluation of Fibularis Tertius Muscle Prevalence. Int J Morphol. 2010;28:759-764.

5. Shigematsu M, Tsubakihara M, Maruyama S. On the m. extensor digitorum longus. Kurume Igakkai Zasshi. 1956;19:1297-1302

6. Bhadkamkar AR, Mysorekar VR. Anomalous peroneus tertius and extensor digitorum longus muscles. J Anat Soc India. 1961;10:37-38.

7. Kelikian AS, Sarrafian SK. Sarrafian's Anatomy of the Foot and Ankle. Lippincott Williams and Wilkins, 2011; p. 122-130.

8. Stevens K, Platt A, Ellis $H$. A cadaveric study of the peroneus tertius muscle. Clin Anat. 1993;6:106-110.

9. Chaney D, Lee M, Khan M. Study of ten anatomical variants of the foot and ankle. J Am Podiat Med Assn. 1996;86:532-537.

10. Plochocki $\mathrm{JH}$, Bodeen M. Unusual supernumerary muscle in the anterior compartment of the leg. Clin Anat. 2010:23:104-105.

11. Banerje A, Singh S, Raza K. Unilateral Variation in Extensor digitorum longus muscle. Clin Ter. 2016;167:150-151.

12. Witvrouw E, Borre KV, Willems TM. The Significance of Peroneus Tertius Muscle in Ankle Injuries. Am J Sports Med. 2006:34:1159-1163.

13. Jungers WL, Medirum D, Stern JT. The functional and evolutionary significance of the human peroneus tertius muscle. J Hum Evol 1993;25:377-386

14. Krammer EB, Lischka MF, Gruber H. Gross anatomy and evolutionary significance of the human peroneus III. Anat Embryol. 1979;155:291-302.

15. Stevens K, Platt A, Ellis H. A cadaveric study of the peroneus tertius muscle. Clin Anat. 1993:6:106-110.

16. Chaney $D$, Lee M, Khan M. Study of ten anatomical variants of the foot and ankle. J Am Podiatr Med Assoc. 1996:86:532-537.

17. Joshi S, Joshi S, Athavale S. Morphology of peroneus tertius muscle. Clin Anat. 2006;19:611-614.

18. Rourke K, Dafydd H, Parkin I. Fibularis tertius: Revisiting the anatomy. Clin Anat. 2007:20:946-949.

19. Kaur AA, Poonam V, Sudhir A. Evaluation of the Origin and Insertion of Extensor Digitorum Longus: Clinical Correlation and Literature Review. JRMEE. 2011;1:50-53.

20. Yildiz S, Yalcin B. An unique variation of the peroneus tertius muscle. Surg Radiol Anat. 2012:34:661-663.

21. Bertelli J, Khoury Z. The peroneus tertius island muscle flap (23.3.91). Surg Radiol Anat. 1991:13:243-244. 